\title{
Investigation of the quenching rate effect on the ferromagnetic properties of $\mathrm{CuO}$ nanoparticles
}

\author{
I.V. Karpov ${ }^{2}$, A.V. Ushakov ${ }^{1,2 *}$, V.G. Demin ${ }^{2}$, E.A. Goncharova ${ }^{2}$, A.A. Shaihadinov ${ }^{2}$ \\ ${ }^{1}$ Federal Research Center Krasnoyarsk Scientific Center, Siberian Branch of the Russian \\ Academy of Sciences, 660036, Krasnoyarsk, Russia \\ ${ }^{2}$ Siberian Federal University, Krasnoyarsk, 660041, Russia \\ *Corresponding author e-mail: ushackov@mail.ru
}

\begin{abstract}
The effect of pressure in a plasma-chemical reactor on the magnetic properties of copper oxide nanoparticles is studied. Plasma-chemical synthesis of copper oxide nanopowders was carried out using an oxygen plasma of an arc discharge at a pressure of 40 and $200 \mathrm{~Pa}$. Transmission electron microscopy (TEM) showed that the obtained sample was highly agglomerated particles with an average particle size of 31 and $27 \mathrm{~nm}$, X-Ray Diffraction (XRD) showed the presence of a single crystalline phase of $\mathrm{CuO}$. Despite the insignificant difference in the average size, the nanoparticles exhibit radically different magnetic behavior. Using a vibrating Superconducting Quantum Interference Device (SQUID) magnetometer, it was shown that the magnetic properties of nanoparticles obtained at $40 \mathrm{~Pa}$ are close in properties to bulk material and exhibit weak ferromagnetism. The nanoparticles obtained at $200 \mathrm{~Pa}$ demonstrate a significant deviation from the properties of the bulk material, show magnetic hardness and shifts in the hysteresis loops.
\end{abstract}

Keywords: Copper oxide; nanoparticles; magnetic properties;

1. Introduction. Copper oxide nanoparticles attract the attention of researchers due to unusual magnetic properties [1,2], which can be widely used in copper-based superconductors [3], highly sensitive sensors [4], as solid electrolytes [5], etc. [6] $\mathrm{CuO}$ is a semiconductor with antiferromagnetic (AFM) ordering. The transition to the paramagnetic state occurs at a Néel temperature $T_{N}=230 \mathrm{~K}$. A detailed study of $\mathrm{CuO}$ nanoparticles obtained by various methods showed that, with a decrease in size, properties such as ferromagnetism (FM) at room temperature (RT) [7], and the effect of exchange bias (EB) [8], temperature change AFM ordering [ibid]. Several models have been proposed to explain these effects. FM was explained by uncompensated spins on the surface of nanoparticles [9], oxygen vacancies causing variable valency. EB was explained by the interaction between the AFM core and the FM shell of uncompensated surface spins. Moreover, the FM shell exhibits spin-glass behavior. A similar effect is also present in $\mathrm{CuO}$ films and nanowires. Initially, the $\mathrm{EB}$ effect was studied exclusively as an interesting phenomenon, but in the last decade, the AFM-FM binary system 
has found its applications in the spin valve and tunnel devices [10]. For a detailed study of this phenomenon, it is necessary to solve one of the most difficult problems - the correct determination of the Néel temperature for $\mathrm{CuO}$ nanoparticles, determined by the behavior of the thermal dependence of susceptibility and magnetization. However, this behavior can be gradual without pronounced peaks. The unusual magnetic behavior of $\mathrm{CuO}$ nanoparticles depends on the magnetic field strength and the production method, which makes it difficult to correctly determine $T_{N}$. Despite this, many authors report a decrease in $T_{N}$ with a decrease in the average size or coherent scattering region of nanoparticles [1].

High-speed quenching of nanoparticles from a plasma state allows one to control the size of nanoparticles from 2 to $40 \mathrm{~nm}$, to create oxygen vacancies, defects, and residual stresses. Previous results on the plasma-chemical synthesis of $\mathrm{CuO}$ nanoparticles showed the presence of FM at RT [11-14]. In this paper, we investigate the morphological and magnetic properties of $\mathrm{CuO}$ nanoparticles obtained at various quenching rates from the plasma state.

2. Experimental. For the synthesis of $\mathrm{CuO}$ nanoparticles, we used an experimental setup described in detail in $[11,12]$. It was shown in [13] that the average nanoparticle size and residual stress correlate with sputtering conditions and depend on the pressure in the vacuum chamber. A physical and mathematical model was proposed to explain such a correlation. The cathode spot emits plasma jets consisting of a microdroplet fraction, clusters, neutral cathode metal atoms, ions and electrons. The quenching of a plasma jet by a buffer gas (this is usually an inert gas) leads to a sharp decrease in the velocity of the plasma jet, heating of the buffer gas, and an increase in the electron temperature of the cathode spot plasma. As a result, the average particle diameter of the microdrop fraction decreases and the processes of plasma chemical synthesis are accelerated, for which reaction gas is introduced into a certain region of the reactor. The quenching rate depends on the rate of diffusion of ions in the plasma jet and the cross section of the elastic collision. The data of the proposed model were interpolated to obtain nanoparticles with an average size of $\sim 25 \mathrm{~nm}$, but with different quenching rates. For plasma-chemical synthesis, an arc evaporator was used with the following characteristics: arc current $100 \mathrm{~A}$, longitudinal magnetic field excited by the focusing coil on the cathode surface, $80 \mathrm{~A} / \mathrm{m}$, distance between the cathode and anode $\mathrm{d}=50 \mathrm{~mm}$. A rod made of high purity copper (99.99\%) with a diameter of $80 \mathrm{~mm}$ and a length of $100 \mathrm{~mm}$ was used as a sputtered cathode. It was installed on a water-cooled copper current lead. Argon was used as the plasma-forming gas, which was supplied through the evaporator and created a base pressure of 40 (sample 1) and $200 \mathrm{~Pa}$ (sample 2) in the chamber. Direct plasma-chemical synthesis was carried out at an oxygen flow rate of 10 vol. \% of the supply of plasma-forming gas. Oxygen was supplied to 
the reactor in such a way as to form a uniform shell around the plasma torch. The morphology of the samples was studied using a JEOL JEM-2100 transmission electron microscope. The crystal structure of the samples was studied using an Advance D8 X-ray diffractometer in $\mathrm{CuK}_{\alpha}$ monochromatized radiation. Quantitative structural-phase analysis of diffractograms was carried out using the Powder Cell 2.4 full-profile analysis program. For identification of XRD spectra, PDF4 + databases were used. Magnetic measurements were carried out using a SQUID magnetometer (Quantum Design). The magnetization curves (dependences $M(H)$ ) were measured after passing the cooling conditions in a zero magnetic field (ZFC). The temperature correlations of the magnetic moment $M(T)$ were measured under ZFC conditions, as well as after cooling in an external field (FC). The temperature varied in the range from 10 to $350 \mathrm{~K}$, an applied measuring field of 500 Oe with subsequent data collection with increasing temperature. To study the effect of exchange bias, the magnetic hysteresis loops $M(H)$ were measured after the sample was cooled from $T=300 \mathrm{~K}$ in zero and nonzero magnetic fields. The exchange bias is characterized by the exchange bias field $H_{E B}=\left(H_{1}-H_{2}\right) / 2$ and the coercive force $H_{C}=\left(H_{1}+H_{2}\right) / 2$, where $H_{1}$ and $H_{2}$ correspond to the values of the magnetic field at which the magnetization of the hysteresis loop changes sign with decreasing and increasing magnetic intensity fields. The shift of the hysteresis loop in FC mode is accompanied by a shift (asymmetry) of the residual magnetization, which is characterized by the similar expressions $M_{E B}=\left(M_{1}-M_{2}\right) / 2, M_{C}=\left(M_{1}+M_{2}\right) / 2$. In this work, the displacement of the hysteresis loops was determined relative to the center of gravity.

3. Results and discussion. Figures 1,2 show TEM images, histograms, and size distribution functions of the size of nanoparticles obtained in an arc discharge plasma at a gas mixture pressure of $90 \% \mathrm{Ar}+10 \% \mathrm{O}_{2} 40$ and $200 \mathrm{~Pa}$.
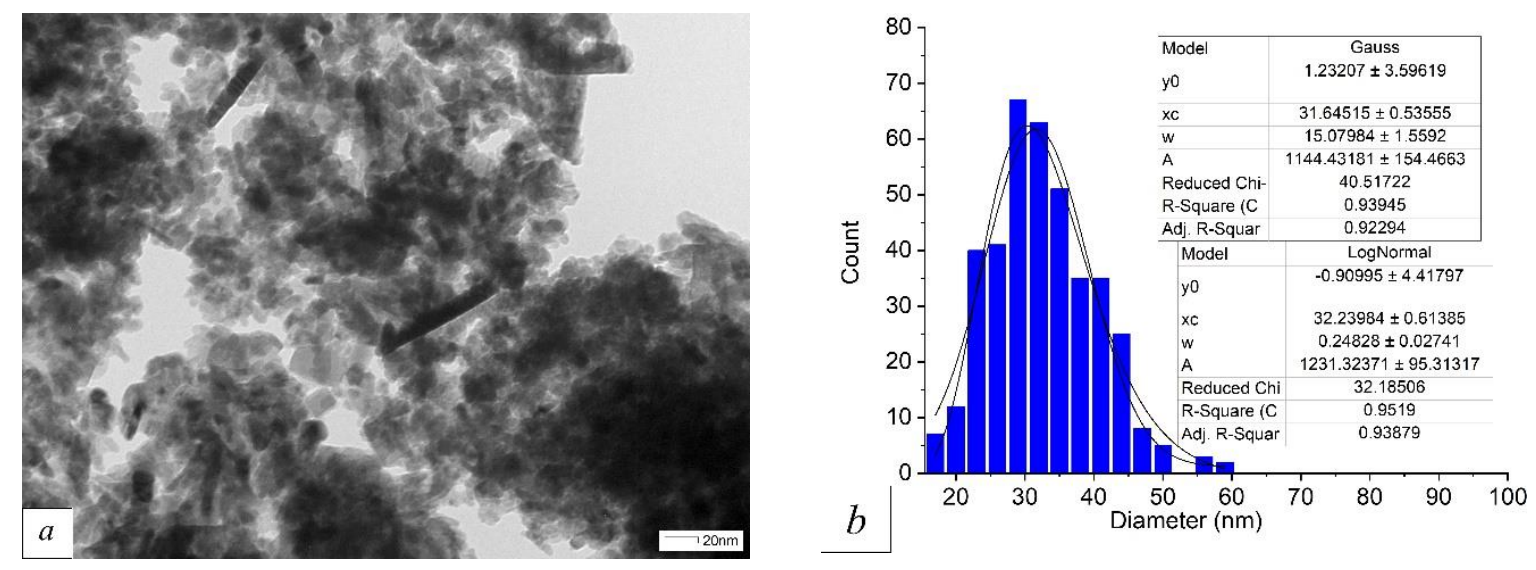

Fig. 1 TEM image (a), histograms and size distribution functions $(b)$ of $\mathrm{CuO}$ nanoparticles obtained in arc discharge plasma at a gas mixture $90 \% \mathrm{Ar}+10 \% \mathrm{O}_{2} 40 \mathrm{~Pa}$ 

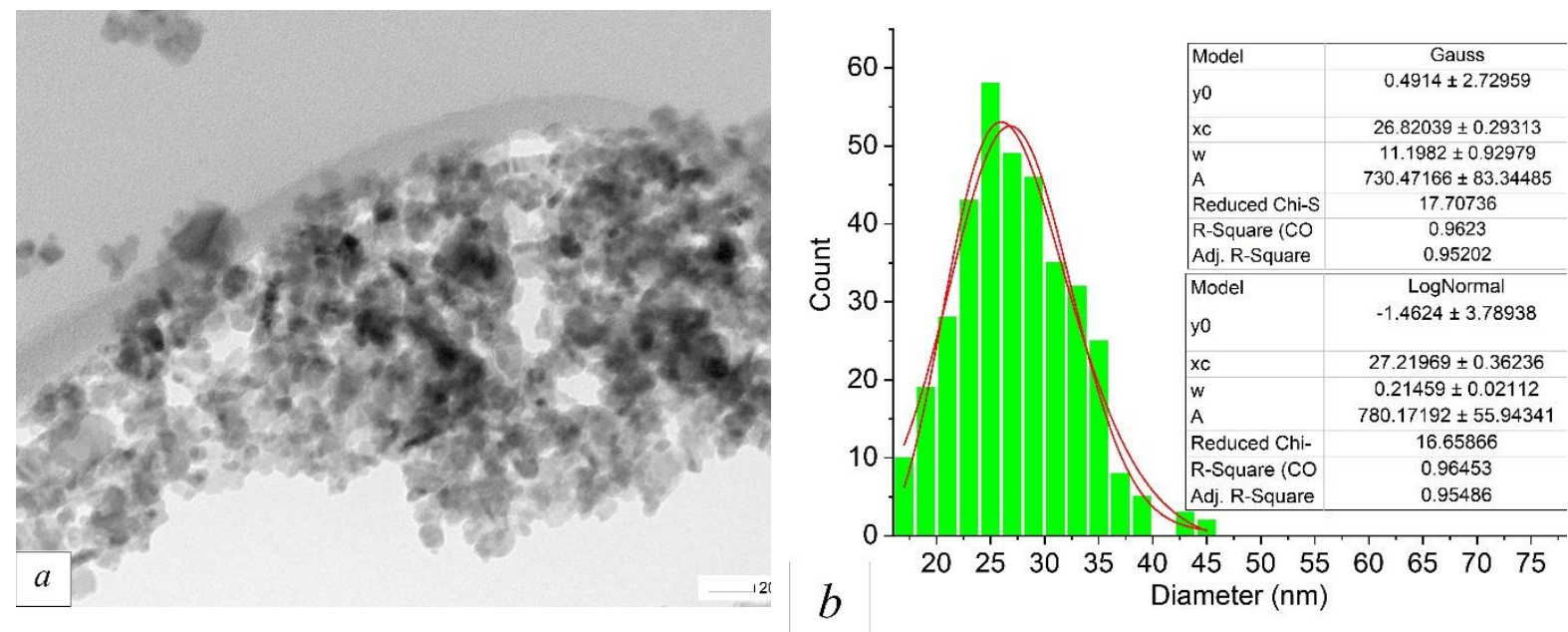

Fig. 2 TEM image (a), histograms and size distribution functions $(b)$ of $\mathrm{CuO}$ nanoparticles obtained in arc discharge plasma at a gas mixture $90 \% \mathrm{Ar}+10 \% \mathrm{O}_{2} 200 \mathrm{~Pa}$

As can be seen in the TEM images, the samples are highly agglomerated particles of almost spherical shape. Plasma-chemical synthesis nanoparticles are characterized by high surface energy; therefore, the particles are highly aggregated, which causes a significant decrease in the specific surface. At a chamber pressure of $40 \mathrm{~Pa}$, the particle sizes range from 15 to $60 \mathrm{~nm}$. The particle size distribution function is directly related to the particle formation process. Particles are characterized by both normal and lognormal particle size distributions. Fitting for both distribution functions gives an average particle size of $32 \mathrm{~nm}$. At a chamber pressure of $200 \mathrm{~Pa}$, the particle sizes range from 15 to $45 \mathrm{~nm}$. Fitting for both distribution functions gives an average particle size of $27 \mathrm{~nm}$. A wider particle size distribution at low pressure and a narrow distribution at high pressure are directly related to the features of plasmachemical synthesis and are discussed in detail in [15-17].

The XRD spectra of the nanopowder obtained at various pressures are shown in Figure 3. The average size of the coherent scattering regions (CSR) and residual crystallite stresses were determined by broadening the spectral lines using full-profile fitting in the PowderCell program. On the XRD spectra, only reflections corresponding to the monoclinic structure of $\mathrm{CuO}$ are present (JCPDS data, No. 45-0937). The results of processing XRD spectra are presented in Table I. 


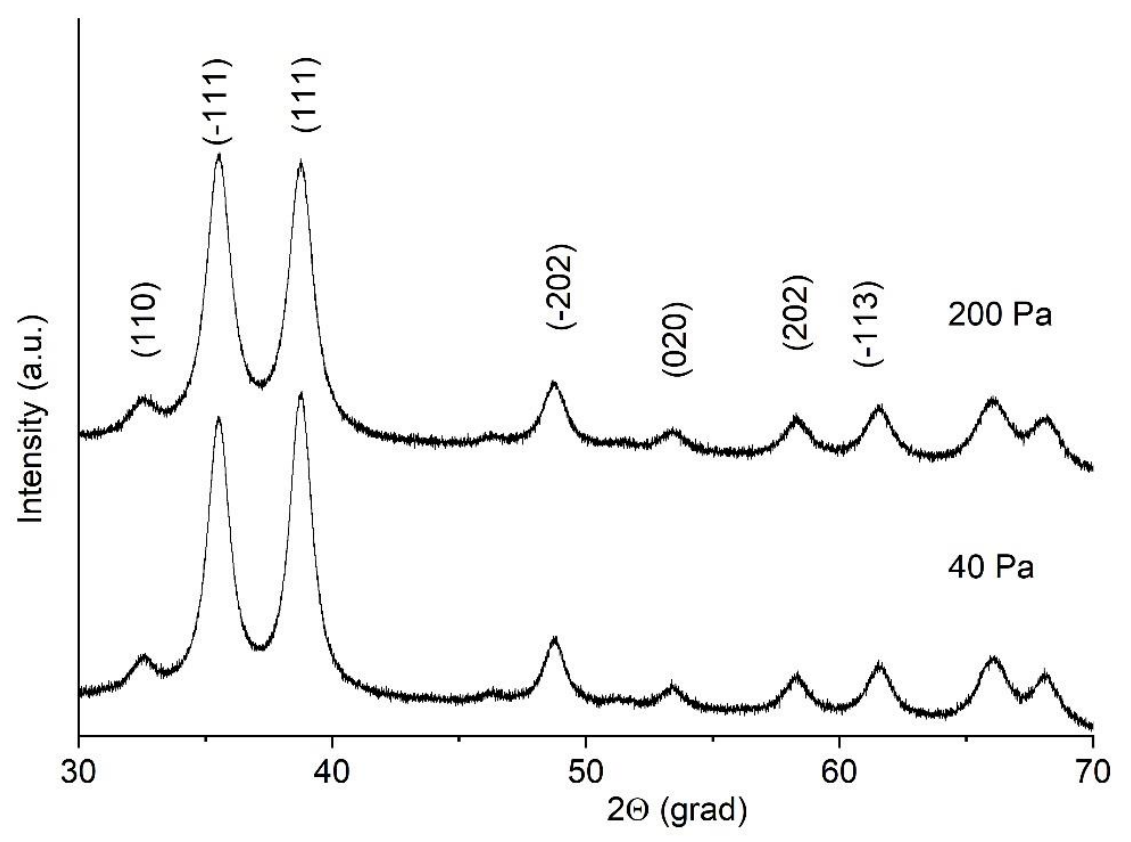

Figure 3. XRD spectra of samples 1 and 2.

Table I. Lattice parameters, average CSR size, residual stresses in $\mathrm{CuO}$ nanoparticles.

\begin{tabular}{|c|c|c|c|c|c|}
\hline Sample & $A(\AA)$ & $b(\AA)$ & $c(\AA)$ & $D(\mathrm{~nm})$ & $\varepsilon(\mathrm{MPa})$ \\
\hline 1 & 4.6764 & 3.4432 & 5.1345 & 29 & 690 \\
\hline 2 & 4.6768 & 3.4441 & 5.1364 & 26 & 720 \\
\hline
\end{tabular}

As follows from the obtained results, an increase in pressure leads to an increase in the rate of quenching of nanoparticles and, as a consequence, to an increase in lattice parameters and residual stresses. The obtained values of the average CSR size are close to the average size of nanoparticles obtained from processing TEM images. An energy-dispersive X-ray spectroscopy (EDX) of samples 1 and 2 showed no contamination.

The temperature dependence of the magnetization of samples 1 and 2 is shown in Fig. 4. As follows from the figure, the irreversibility between the dependences $M_{\mathrm{ZFC}}(T)$ and $M_{\mathrm{FC}}(T)$ begins above RT and is estimated at $T_{I r} \sim 350 \mathrm{~K}$ for both samples. However, the behavior of the $M_{\mathrm{ZFC}}(T)$ and $M_{\mathrm{FC}}(T)$ curves for samples 1 and 2 is different. The behavior of the $M_{\mathrm{ZFC}}(T)$ and $M_{\mathrm{FC}}(T)$ curves for sample 1 obtained at a gas mixture pressure of $40 \mathrm{~Pa}$ almost coincides with the curves for a bulk $\mathrm{CuO}$ sample. In contrast to sample 1, the $M_{\mathrm{ZFC}}(T)$ and $M_{\mathrm{FC}}(T)$ curves for sample 2 do not show peaks characteristic of the bulk sample. For $M_{\mathrm{FC}}(T)$, there is a broadened peak at $T \sim 100 \mathrm{~K}$ and a monotonic increase with decreasing temperature, the $M_{\mathrm{ZFC}}(T)$ curve shows a plateau for temperatures in the range 40-140 K. The inset shows the difference in the susceptibilities $\left.\left(M_{\mathrm{ZFC}}(T)-M_{\mathrm{FC}}(T)\right)\right)$ at 500 and 100 Oe. You can see a 
monotonous increase with decreasing temperature. The obtained dependences make it possible to determine the temperature of the antiferromagnetic ordering $T_{N}$ by the peaks on the curves $(\partial \chi(T)) / \partial T$, where $\chi(T)$ is the susceptibility at a magnetic field of $H=500$ Oe. The obtained value of $195 \mathrm{~K}$ for sample 1 , which is lower than for a bulk sample, agrees well with that obtained previously by other authors [1]. The authors attribute this phenomenon to a decrease in the energy of the exchange interaction associated with the quantum-size effect, oxygen vacancies, etc. For sample 2, the calculations give two maxima at 30 and $120 \mathrm{~K}$, which indicates the complex nature of magnetic ordering. The temperature dependence of the magnetization of sample 2 was studied at a field strength of 100 Oe. In this field, the irreversibility between the $M_{\mathrm{ZFC}}(T)$ and $M_{\mathrm{FC}}(T)$ dependences starts above $350 \mathrm{~K}$. The $M_{\mathrm{ZFC}}(T)$ curve has two broad maxima at 80 and $150 \mathrm{~K}$, and the derivative $(\partial \chi(T)) / \partial T$ has a maximum at $50 \mathrm{~K}$. Thus, with increasing magnetic field strength, $T_{N}$ shifts to the low-temperature region.

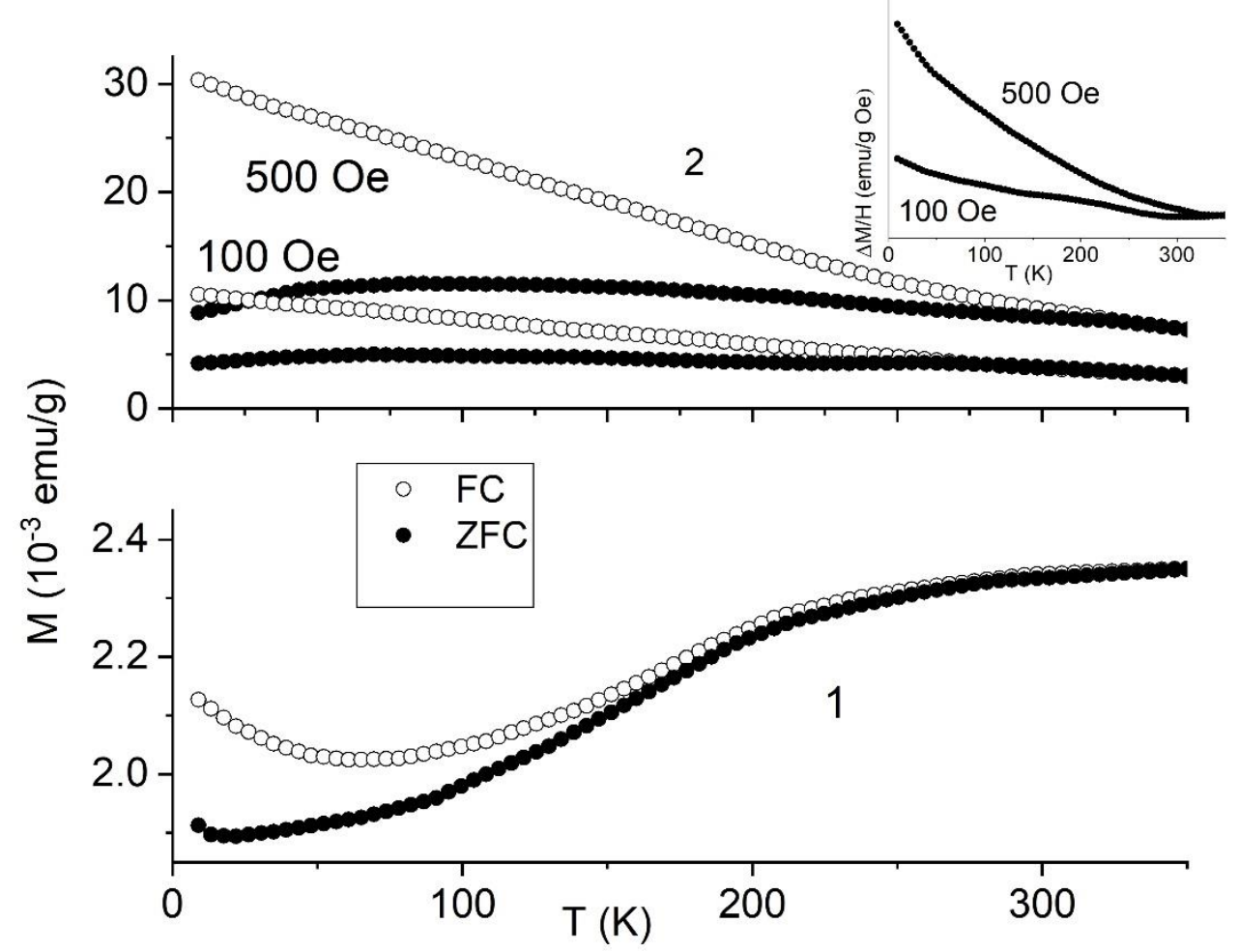

Figure 4. Temperature dependence of the magnetization $M_{\mathrm{ZFC}}(T)$ and $M_{\mathrm{FC}}(T)$ of samples 1 and 2 at a magnetic field strength of 500 and 100 Oe. The inset shows the difference dependence of the susceptibilities $\left(M_{\mathrm{FC}}(T)-M_{\mathrm{ZFC}}(T)\right)$ at 500 and 100 Oe for sample 2.

In $[1,14]$, the magnetization of $\mathrm{CuO}$ nanoparticles with a nominal size of 37-6.6 nm obtained by the sol-gel method and $12 \mathrm{~nm}$ obtained by the plasma chemical method was studied. It was shown that nanoparticles $\geq 10 \mathrm{~nm}$ [1] behave as bulk $\mathrm{CuO}$, taking into account an increase 
in the lattice parameter. However, for nanoparticles $\leq 10 \mathrm{~nm}$ [1] and $12 \mathrm{~nm}$ [14], the calculated magnetic moment agrees with the Néel model, taking into account the uncompensated magnetic moments of alternating sublattices of the surface layer. For such particles, a magnetic transition is observed and the appearance of anomalous hysteresis loops at temperatures below 40K. There is a sharp increase in $M_{\mathrm{r}}$ and $H_{\mathrm{c}}$. This temperature is the Néel temperature of the transition to the magnetic state with the exchange interaction of uncompensated magnetic spins of the shell and spins of the $\mathrm{Cu}^{2+}$ core. For sample 2, it would be logical to assume a bimodal particle size distribution, however, a careful study of HRTEM images does not confirm this assumption. Since in the plasma-chemical synthesis the crystallization process has an avalanche-like character, the nanoparticles have a dendritic structure with a predominant growth towards the evaporator [12]. Uniaxial magnetic anisotropy can be associated precisely with the dendritic structure of nanoparticles. The diameter of an individual dendrite reaches a cluster size of $\leq 2$ $\mathrm{nm}$. Such structures are clearly visible on the XRD spectra in the form of broadening of the base of peaks or halo. Oxygen stoichiometry can also vary widely from deficit to excess, depending on the thermal state of the substrate. Moreover, the transition from the crystalline core of the nanoparticle to the dendritic shell is smooth.

Figure 5 shows the field dependence of the magnetization of samples $1,2 \mathrm{CuO}$ at $\mathrm{RT}$. As follows from the figure, both curves demonstrate ferromagnetism at low field strengths. The coercive force $H_{C}$ was 40 and $300 \mathrm{Oe}$, and the residual magnetization $M_{\mathrm{r}}$ was $1.7 \cdot 10^{-4}$ and $6.2 \cdot 10^{-3} \mathrm{emu} / \mathrm{g}$ for samples 1 and 2 , respectively.

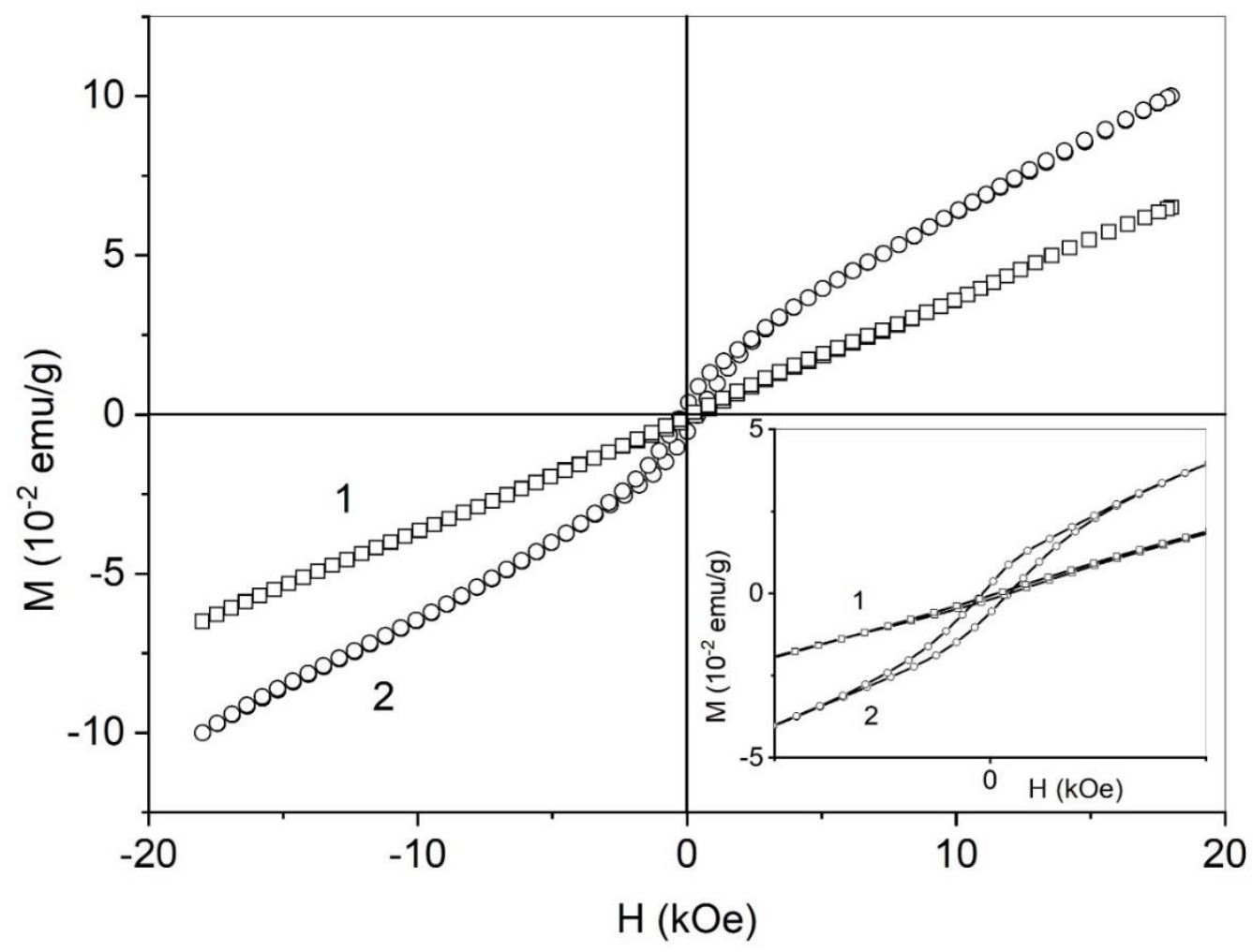


Figure 5. Field dependence of the magnetization of samples 1, $2 \mathrm{CuO}$ at RT. The inset shows hysteresis loops.

In strong fields, the loops do not reach saturation and exhibit an almost linear dependence. After simple calculations, we can subtract the linear contribution and determine the saturation magnetization, which amounted to $1.3 \cdot 10^{-3}$ and $2.8 \cdot 10^{-2} \mathrm{emu} / \mathrm{g}$ for samples 1 and 2 , respectively. The slope of the linear contribution indicates the paramagnetic component of the core of the nanoparticles.

To study the temperature dependence of the ferromagnetism of the obtained $\mathrm{CuO}$ nanoparticles, we studied the field dependences of the magnetization under ZFC and FC conditions at a temperature of $40 \mathrm{~K}$, which are shown in Fig. 6. The curves show only the ferromagnetic contribution to the magnetization. At low temperatures, the linear contribution to the magnetization remains the most significant in the total magnetization.

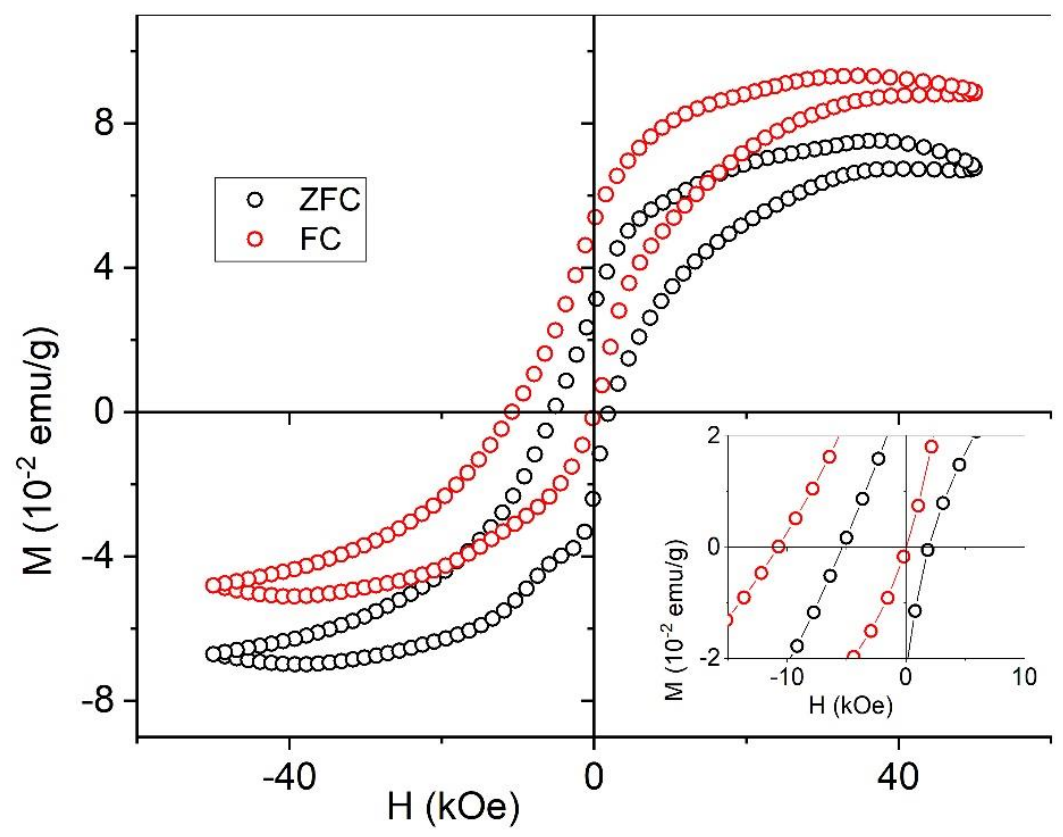

Figure 6. Hysteretic dependences of the magnetization of sample $2 \mathrm{CuO}$ in the $\mathrm{FC}$ and ZFC modes at a temperature of $40 \mathrm{~K}$. The inset shows exchange bias effect.

Comparing the hysteresis loops, you can see that the FC loop is wider than $\mathrm{ZFC}, H_{\mathrm{ZFC}}=$ $2350 \mathrm{Oe}, H_{\mathrm{FC}}=2730 \mathrm{Oe}$. An interesting feature of the loops is the increase in width in stronger fields. This feature suggests the presence of nanoparticles with different magnetic hardness [18] or the presence of superparamagnetic particles [19]. Residual magnetization $M_{\mathrm{rZFC}}=3.1 \cdot 10^{-2}$ Oe, $M_{\mathrm{rFC}}=4.4 \cdot 10^{-2}$ Oe. In addition, the FC loop is shifted up and to the left, which clearly indicates the $\mathrm{EB}$ effect and the estimate gives $H_{\mathrm{EB}}=1100$ Oe. 
In [20] the authors found a similar behavior for $\mathrm{NiO}$ nanoparticles in the field dependence of $M(H)$ at $5 \mathrm{~K}$. The new effect is explained by the small particle size and the significant contributions of surface atoms. Impaired coordination of surface spins causes a change in magnetic ordering throughout the particle. The large coercivity and the shift of the loops are satisfactorily explained by the weak coupling between the sublattices and the different spin paths when the field is applied. The authors constructed model curves and explained the changes in magnetic order over the entire particle by decreasing the length of structural coherence.

The $\mathrm{EB}$ effect in $\mathrm{CuO}$ nanoparticles appears as a result of exchange interactions of the AFM core and shell with uncompensated surface spins responsible for the appearance of FM at RT. The shell is formed as a result of high-speed deposition of $\mathrm{CuO}$ clusters from the plasma phase onto the surface of the "core" of the nanoparticle. As a result, a dendritic structure with a high specific surface [21] is formed. The specific surface of sample 2, measured by the BET method, was $130 \mathrm{~m}^{2} / \mathrm{g}$. XRD studies also showed a large value of residual stresses, which also affects the FM of nanoparticles [13]. Due to the large specific surface, a layer of sorbed gases is formed on the surface of the nanoparticles, which also affects the magnetization of the nanoparticles.

\section{Conclusion}

As a result of the evaporation of the copper cathode in oxygen plasma of a low-pressure arc discharge, single-phase $\mathrm{CuO}$ nanoparticles with an average size of 32 (sample 1) and $27 \mathrm{~nm}$ (sample 2) are formed, depending on the pressure of the gas mixture in the plasma chemical reactor. All samples demonstrate magnetic irreversibility above RT and FM in weak fields. Despite the insignificant difference in the average size, the magnetic behavior of sample 1 is similar to the bulk material, but the Néel temperature was $195 \mathrm{~K}$, which is significantly lower, and sample 2 does not show the peaks characteristic of the bulk sample (the broadened peak is visible on the $M_{\mathrm{FC}}(T)$ curve at $T \sim 100 \mathrm{~K}$ ) and, accordingly, the determination of the Néel temperature is difficult. At a low temperature $(40 \mathrm{~K})$ in sample 2, the FC hysteresis loops are displaced, which may indicate the EB effect.

Acknowledgements. The work was performed with a support of the grant of the Russian Science Foundation (Project No. 16-19-10054). 


\section{References}

1. A. Punnooose, H. Magnone, M.S. Seehra, Phys. Rev. B, 64, 174420, (2001).

2. D. Lisjaka, A. Mertelj, Progress in Mater. Sci., 95, 286, (2018).

3. A.V. Ushakov, I.V. Karpov, A.A. Lepeshev, M.I. Petrov, J. Appl. Phys. 118, 023907, (2015).

4. I. Safarik, M. Safarikova, Mater. Sci. Eng., 133, 737, (2002).

5. O. Waser, M. Hess, A.T. Güntner, P. Novák, S.E Pratsinis, J. of Power Sources, 241, 415, (2013).

6. Q. Zhang, K. Zhang, D. Xu, G. Yang, H. Huang, F. Nie, C. Liu, S. Yang, Progress in Mater. Sci., 60, 208, (2014).

7. H. Qin, Z. Zhang, X. Liu, Y. Zhang, J. Hu, JMMM, 322, 1994, (2010).

8. X.G. Zheng, T. Moria, K. Nishiyama, W. Higemoto, C.N. Xu, Solid State Commun., 132, 493, (2004).

9. F.D. Fischer, T. Waitz, D. Vollath, N.K. Simha, Progress in Mater., Sci., 53, 481, (2008).

10. S.K. Giri, T.K. Nath, J. Nanosci. Nanotechnol., 14, 1209, (2014).

11. I.V. Karpov, A.V. Ushakov, A.A. Lepeshev, L.Yu. Fedorov, Tech. Phys., 62, 168, (2017).

12. A.V. Ushakov, I.V. Karpov, A.A. Lepeshev, J of Supercond. and Novel Magn., 30, 311, (2017).

13. I.V. Karpov, A.V. Ushakov, V.G. Demin, A.A. Shaihadinov, A.I. Demchenko, L.Yu. Fedorov, E.A. Goncharova, A.K. Abkaryan, JMMM, 490, 165492, (2019).

14. A.V. Ushakov, I.V. Karpov, A.A. Lepeshev, J of Supercond. and Novel Magn., 30, 3351, (2017).

15. Aghdam H. Dizajghorbani, H. Azadi, M. Esmaeilzadeh, S. Moemen Bellah, R. Malekfar, Optical Mater., 91, 433, (2019).

16. I.V. Karpov, A.V. Ushakov, L.Yu. Fedorov, E.A. Dorozhkina, O.N. Karpova, A.A. Shaikhadinov, V.G. Demin, A.I. Demchenko, M.V. Brungardt, E.A. Goncharova, Phys. of the Solid State, 61, 1180, (2019).

17. A.H.M. Al-Antaki, X. Luo, X. Duan, R.N. Lamb, W.D. Hutchison, W. Lawrance, C.L. Raston, ACS Omega, 4, 13577, (2019).

18. L.H. Bennett, E. Della Torre, J of Appl. Phys., 97, 10E502, (2005).

19. L. Tauxe, T.A.T. Mullender, T. Pick, J of Geophys. Res., 101, 571, (1996).

20. R.H. Kodama, S.A. Makhlouf, A.E. Berkowitz, Phys. Rev. Lett., 79, 1393, (1997). 
21. A.A. Lepeshev, G.S. Patrin, G.Yu. Yurkin, A.D. Vasiliev, I.V. Nemtsev, D.M. Gokhfeld, A.D. Balaev, V.G. Demin, E.P. Bachurina, I.V. Karpov, A.V. Ushakov, L.Yu. Fedorov, L.A. Irtyugo, M.I. Petrov, J of Supercond. and Novel Magn., 31, 3841, (2018). 